\title{
Role of the Carrier-Envelope Offset Phase of Few-Cycle Pulses in Nonperturbative Resonant Nonlinear Optics
}

\author{
O. D. Mücke, T. Tritschler, and M. Wegener \\ Institut für Angewandte Physik, Universität Karlsruhe (TH), Wolfgang-Gaede-Straße 1, 76131 Karlsruhe, Germany \\ U. Morgner and F. X. Kärtner \\ Institut für Hochfrequenztechnik und Quantenelektronik, Universität Karlsruhe (TH), \\ Engesserstraße 5, 76131 Karlsruhe, Germany \\ (Received 11 January 2002; published 28 August 2002)
}

\begin{abstract}
We study the influence of the carrier-envelope offset phase of few-cycle pulses on nonperturbative resonant extreme nonlinear optics in a semiconductor. If the Rabi frequency becomes comparable to the light frequency, the different Rabi sidebands interfere around twice the laser center frequency, giving rise to a signal which strongly depends on the carrier-envelope offset phase. This signature should be measurable in GaAs samples.
\end{abstract}

DOI: $10.1103 /$ PhysRevLett.89.127401

PACS numbers: 78.47.+p, 42.50.Md, 42.65.Re

The rapid development of yet shorter and shorter laser pulses [1] has now led us into a regime in which the phase between the rapidly oscillating light frequency and the electric field envelope has become a relevant quantity [2-4]. This carrier-envelope offset (CEO) phase, $\phi$, of a single few-cycle pulse can significantly influence the outcome of an experiment. It has to be distinguished from the well-known relative optical phase, i.e., the phase between two different beams or pulses.

In order to fix the CEO frequency $f_{\phi}$, recent work $[5,6]$ has, for example, used the interference of the fundamental frequency of a laser pulse, which was spectrally broadened by self-phase modulation in a (photonic crystal) fiber of a few millimeter length, with the second harmonic generated with the help of a separate crystal. In Ref. [7], the same idea was used, except for the fact that the fundamental spectrum did already cover one octave, hence no need for additional broadening. Somewhat similar to this, Ref. [8] proposed to use the interference of the third harmonic, generated at a silicon wafer surface, with the second harmonic generated in a separate crystal. All the optical nonlinearities used in these and other [9] cases are off-resonant and within the perturbative regime; i.e., an expansion in terms of nonlinear optical susceptibilities is meaningful. Furthermore, in most of these cases, the pulses have to propagate over a considerable distance within the apparatus and, hence, the difference between the phase velocity and the group velocity can change the CEO phase within the measurement setup. Currently, this is the main obstacle in measuring $\phi$.

A nonperturbative and, hence, distinctly different way to determine the CEO phase would be via $\mathrm{x}$-ray generation in extreme nonlinear optics in atoms $[1,10]$. In a recent review [11] on the implications of the CEO phase on metrology $[12,13]$, the authors state in their outlook: "... If an experimental technique can be developed that is sensitive to the carrier-envelope phase and works with the direct output of a mode-locked oscillator (i.e., without that amplification that will be necessary for extreme nonlinear optics), it may in turn benefit optical-frequency synthesis because it may create a simpler technique for determining/controlling the comb offset frequency."

In this Letter, we show that resonant extreme nonlinear optics in a solid, exemplified by carrier-wave Rabi flopping [14], shows a dependence on the CEO phase $\phi$, which potentially allows one to determine $\phi$. The idea: For conventional envelope Rabi flopping, the oscillation of the inversion with Rabi frequency $\Omega_{R}$ modulates the optical polarization. This leads to a pair of sidebands, separated by $\Omega_{R}$, centered around the optical transition frequency (equal to the laser center frequency $\omega_{0}$ ). This is the resonant analog of self-phase modulation. Beyond the rotating wave approximation, one additionally finds similar pairs of sidebands around all the uneven harmonics of the laser center frequency. If, for example, the Rabi frequency is twice as large as the laser center frequency, the high-energy peak of the fundamental pair and the low-energy peak of the third-harmonic pair meet at around twice the laser center frequency [see inset in Fig. 1(a)]. In analogy to the above-mentioned off-resonant approaches, this interference is expected to strongly depend on the CEO phase. The required coherence of the two-level system as well as the necessary extreme electric field amplitudes (for a solid) can be achieved by strongly focused few-cycle pulses.

Early experiments [14], however, showed a much smaller splitting of the sidebands than expected from a modeling [14-16] based on an ensemble of identical twolevel atoms, embedded in a material with a background dielectric constant. As a result of this small splitting, the above interference around twice the laser center frequency is strongly reduced, questioning whether any influence of the CEO phase could be detected within this scheme. 


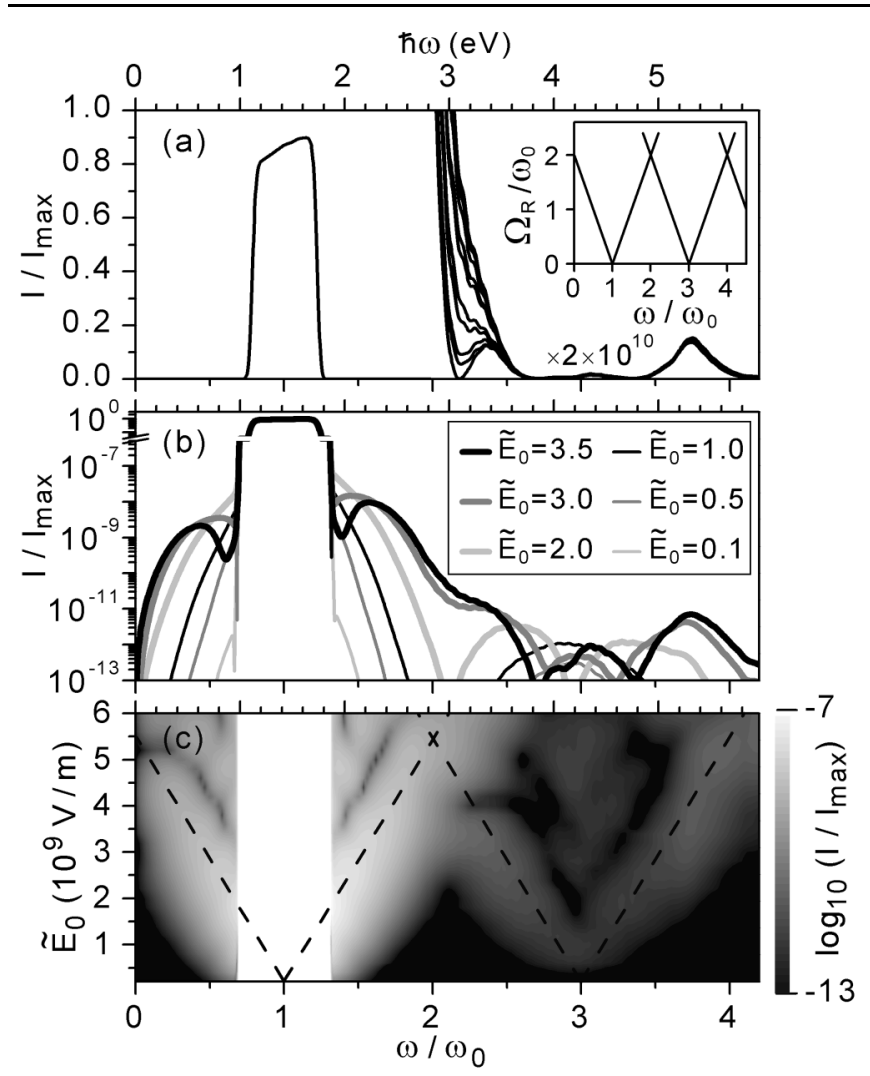

FIG. 1. (a) Signal intensity (normalized to the maximum intensity, $I_{\max }$, of the incident laser spectrum) emitted into the forward direction versus spectrometer frequency $\omega$ in units of the laser center frequency $\omega_{0}$ for different values of the CEO phase, $\phi=0, \ldots, \pi$. The GaAs film with $L=20 \mathrm{~nm}$ thickness on a substrate with $\epsilon_{\mathrm{s}}=\mathrm{const}=(1.76)^{2}$ has no $\mathrm{Al}_{0.3} \mathrm{Ga}_{0.7} \mathrm{As}$ barriers on either side, but a frontside antireflection coating, $\tilde{E}_{0}=3.5 \times 10^{9} \mathrm{~V} / \mathrm{m}$. The inset illustrates the interference of peaks from the different pairs of Rabi sidebands as the Rabi frequency $\Omega_{\mathrm{R}}$ increases. (b) As (a), but signal intensity (normalized) for fixed $\phi=0$ and for different incident electric field amplitudes (in units of $10^{9} \mathrm{~V} / \mathrm{m}$ ) as indicated. (c) As (b), but grey-scale image. The dashed lines are guides to the eye.

In what follows we (i) motivate and (ii) define the model, (iii) discuss an experimentally accessible situation in which the above-mentioned effects arising from the CEO phase do occur indeed, and (iv) apply the exact same theory with the same material parameters to previous experiments performed on other sample designs in order to test the validity of our approach.

(i) Motivation of the model.-The simple modeling [14-16] has several shortcomings when applied to semiconductors: (1) It lumps all transitions at high energies into a background dielectric constant and (2) usually, (quantum kinetic) Coulomb correlations can also have a strong influence on actual experiments. Which of the two is more important depends on the sample design. While, e.g., propagation effects associated with (1) can, in principle, be minimized by choice of sample design, we will see that this limit is only strictly reached for freestanding
$10 \mathrm{~nm}$ thin, both sides antireflection-coated sampleswhich may be difficult to realize in practice. A combined theoretical treatment of (1) and (2) is currently not in reach. Let us have a closer look at these aspects. (1) The concept of the background dielectric constant implies an instantaneous screening of the external laser field. Is this justified under the conditions of this Letter? The dominant contributions to the background dielectric constant in the model semiconductor GaAs [17] stem from the known $E_{1}$ and $E_{2}$ resonances at 3 and $5 \mathrm{eV}$ photon energy, respectively. This results in a detuning with respect to the band edge of about $1.5 \mathrm{eV}\left(E_{1}\right)$, which is equivalent to a period of about $2.7 \mathrm{fs}$. This is comparable to a cycle of light (2.9 fs at the GaAs band gap) and cannot be considered as instantaneous. This finite response time of the dielectric screening leads to distortions of the incident pulses within the sample (if and only if propagation effects are accounted for) and related effects which can altogether modify the experimental results substantially. (2) Excitonic effects lead to a very large enhancement of optical nonlinearities near the band gap. This enhancement makes the modeling based on identical two-level systems [14-16] at the band gap meaningful in the first place. Also, Coulomb correlations strongly influence the shape of the linear dielectric function up to several $\mathrm{eV}$ photon energy, a point which will effectively be accounted for in (ii). Another aspect is the renormalization of the Rabi energy, in which the sum over interband transition amplitudes (the modulus of which is limited by 1) times the Coulomb interaction, the "internal" Rabi energy, adds to the Rabi energy of the laser pulses [18]. However, as the contribution of high-energy transitions decreases with increasing energy because of stronger dephasing, the internal contribution is limited in magnitude. In particular, it is not expected to become comparable to the band gap energy, which is on the order of the Rabi energy of the laser pulses for the conditions of carrier-wave Rabi flopping. This is also confirmed in calculations accounting for these effects, but neglecting propagation [19]. The role of quantum kinetic memory effects in extreme nonlinear optics could be the subject of interesting future studies.

(ii) Definition of the model.-We start from the wellknown Bloch equations for a semiconductor [18] with fixed phenomenological dephasing times, $T_{2}$, coupled to the Maxwell equations, assume one-dimensional propagation (in the $z$ direction) and neglect the Coulomb interaction among carriers. While calculations like that have been done numerous times for transitions close to the band edge, optical transitions high in the bands have rarely been accounted for. In order to do this, one needs to account for the actual band structure (i.e., one must not employ the effective mass approximation). Difficulties do arise from the fact that the optical dipole matrix element strongly depends on the electron wave vector-which again needs accurate band structure calculations. We 
avoid all these challenging problems by taking advantage of the fact that the high-energy optical transitions do not contribute significantly anyway to the nonlinear signals due to a huge resonant enhancement at the band edge as discussed in Refs. $[14,20]$. Thus, we neglect the occupation of these high-energy transitions and determine the distribution of this ensemble of two-level systems by a fit to the accurately measured linear complex dielectric function [17]. This is advantageous because theory was never able to provide a true quantitative match to measurements of the linear dielectric function; however, the actual quantitative shape of the dielectric function is very important for what follows. The only optical nonlinearity in our model arises from those two-level transitions at the GaAs $\left(\mathrm{Al}_{0.3} \mathrm{Ga}_{0.7} \mathrm{As}\right)$ band edge at $E_{\mathrm{g}}=1.42 \mathrm{eV}(1.83 \mathrm{eV})$ photon energy (their dipole matrix element is $d=$ $0.5 e \mathrm{~nm}$, their density $10^{18} \mathrm{~cm}^{-3}, T_{2}=20,30$, or $50 \mathrm{fs}$ deliver indistinguishable results, $T_{1}=\infty$ ). The resulting linear dielectric function, fitted by means of 45 two-level systems, is shown in Fig. 3(b). A crucial point in our modeling is that we account for the actual sample geometry, i.e., including all the layers of the sample. All these aspects together with the fact that we do not employ the slowly varying envelope approximation and do not employ the rotating wave approximation make this a demanding numerical task. For clarity, we show in the following results for optical pulses with $E(t)=$ $\tilde{E}_{0} \operatorname{sinc}\left(t / t_{0}\right) \cos \left(\omega_{0} t+\phi\right)$ which roughly resemble recent experiments [14] [where $\operatorname{sinc}(x)=\sin (x) / x$ ]. The full width at half maximum of the intensity is $5.6 \mathrm{fs}$ (i.e., $t_{0}=5.6 \mathrm{fs} / 2.7831$ ), the center photon energy $\hbar \omega_{0}=$ $1.42 \mathrm{eV}=E_{\mathrm{g}}$.

(iii) Influence of the CEO phase in thin GaAs samples.-Figure 1(a) shows the spectra of light emitted into the forward direction of a thin layer of GaAs which has no $\mathrm{Al}_{03} \mathrm{Ga}_{07}$ As barriers for various values of the CEO phase $\phi$. Such samples can be produced by molecular-beam epitaxy on GaAs substrates and subsequent selective etching, first of the GaAs substrate and then of the $\mathrm{Al}_{0.3} \mathrm{Ga}_{0.7} \mathrm{As}$ etch stop layer. The inset schematically illustrates the meeting of different Rabi sidebands as discussed above. This behavior can also be seen in the actual calculations [Figs. 1(b) and 1(c)]. Furthermore, it becomes clear from the intensity dependence shown in Fig. 1(b) that it is not simply the interference of the tail of the laser spectrum itself with the third-harmonic signal-which would be similar to all the approaches outlined in the introduction-but rather the interference of different Rabi sidebands.

Figure 2(a) depicts the intensity spectra of light emitted into the forward direction versus CEO phase $\phi$ for a $L=100 \mathrm{~nm}$ thin GaAs film on a substrate with $\epsilon_{\mathrm{s}}=$ $(1.76)^{2}$ (e.g. sapphire). Note the dependence on $\phi$ with large visibility around $\omega / \omega_{0}=2.05-2.25$ (this is a $284 \mathrm{meV}$ or $38 \mathrm{~nm}$ broad interval) and the period of $\pi$ (rather than $2 \pi$ for Refs. [5-9]) resulting from the inver- sion symmetry of the problem [21]. Figure 2(b) shows the same for $L=20 \mathrm{~nm}$, indicating that one already has some distortions in 2(a) due to the finite thickness of the sample as a result of different group and phase velocities [we come back to these distortions in section (iv)]. Below $L=20 \mathrm{~nm}$, these propagation effects are negligible. Figure 2(c) is as 2(b), but introducing a frontside $\lambda / 4$-antireflection coating designed for the fundamental laser center frequency $\omega_{0}$. (In an actual antireflection coating, the small dispersion of the dielectric might lead to minor modifications.) Note that 2(b) and 2(c) are shifted with respect to each other horizontally, because the incident optical pulses, and thus also $\phi$, are distorted as a result of multiple reflections. Figure 2(d) is as 2(c), but for a different incident electric field amplitude $\tilde{E}_{0}$. This variation also leads to a horizontal shift, which is both interesting as well as disturbing. It is interesting, on the one hand, because no such intensity dependence occurs in off-resonant perturbative nonlinear optics, following Ref. [8] - pointing out the distinct difference between the two scenarios. It is disturbing, on the other hand, because in order to use the effect to determine the CEO phase, one needs to calibrate the incident electric field amplitude, or, more precisely, the Rabi frequency. This is, however, possible via the measured splitting of the Rabi sidebands. Finally, it is important to note that the excitation intensities (or field amplitudes) and the absolute emitted signals shown here are comparable to the signals computed for samples corresponding to previous experiments [14] [see section (iv)] in which the nonlinear signals could easily be detected using pulses with $81 \mathrm{MHz}$

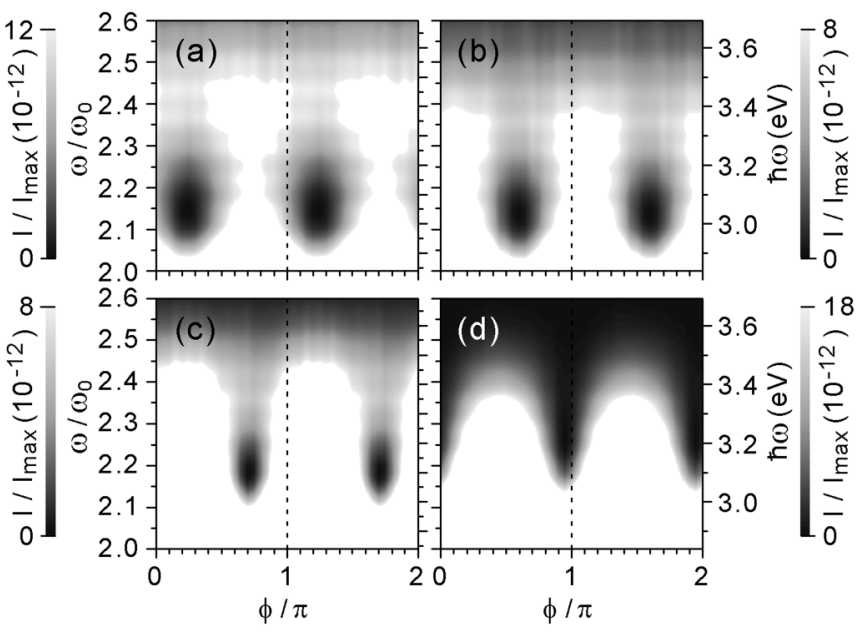

FIG. 2. Grey-scale image of the emitted intensity as a function of $\omega$ and $\phi$ for a thin GaAs film with thickness $L$ without $\mathrm{Al}_{0.3} \mathrm{Ga}_{0.7} \mathrm{As}$ barriers on a substrate with dielectric constant $\epsilon_{\mathrm{s}}$. (a) $L=100 \mathrm{~nm}, \tilde{E}_{0}=3.5 \times 10^{9} \mathrm{~V} / \mathrm{m}$, and $\epsilon_{\mathrm{s}}=(1.76)^{2}$; (b) as (a) but $L=20 \mathrm{~nm}$; (c) as (b) but with an additional frontside antireflection coating (as in Fig. 1); (d) as (c) but for an electric field amplitude of $\tilde{E}_{0}=4.0 \times 10^{9} \mathrm{~V} / \mathrm{m}$. 


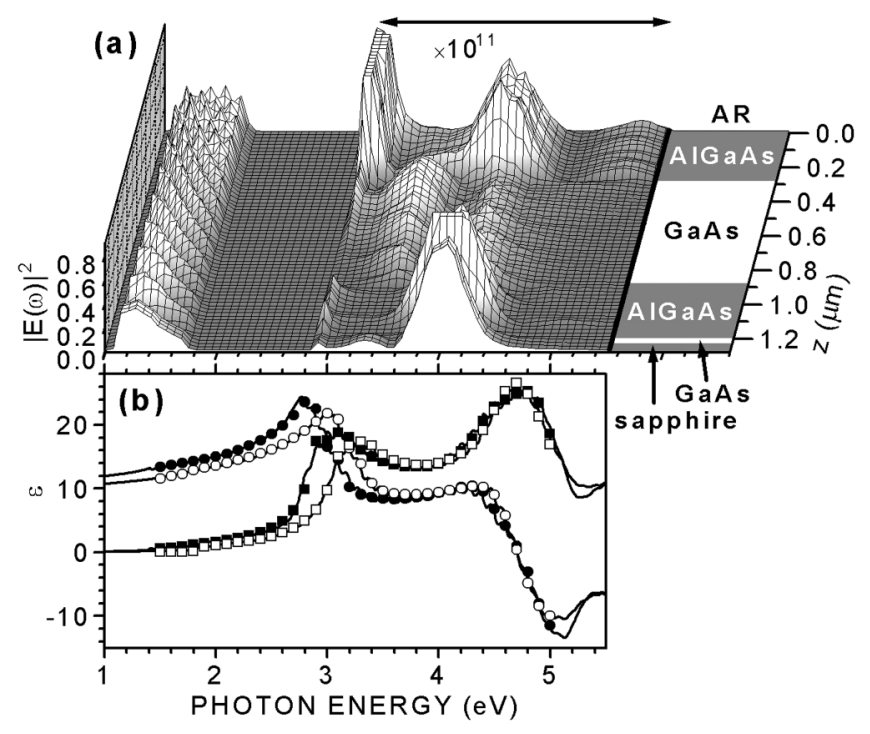

FIG. 3. (a) Calculations for a GaAs double heterostructure as the one used in Ref. [14]. $|E(\omega)|^{2}$ (linear scale, normalized) as a function of $\hbar \omega$ and propagation coordinate $z, \tilde{E}_{0}=3.5 \times$ $10^{9} \mathrm{~V} / \mathrm{m}, \phi=0$. The peak of the emitted signal around the third harmonic corresponds to an intensity of $I / I_{\max }=16 \times$ $10^{-12}$ which is comparable to the values shown in Figs. 1 and 2. Note the strong variation as a function of $z$. (b) The real (circles) and imaginary (squares) part of the linear dielectric function of $\mathrm{GaAs}$ (solid) and $\mathrm{Al}_{0.3} \mathrm{Ga}_{0.7}$ As (open), respectively, are shown for comparison. The symbols are the experimental data taken from Ref. [17]; the full curves correspond to our modeling.

repetition rate directly out of a laser oscillator with average powers of merely $10 \mathrm{~mW}$.

(iv) Consistency check with previous experiments on a GaAs $/ A l_{0.3} G a_{0.7}$ As double heterostructure.-In early experiments [14], a different sample design-a GaAs double heterostructure - was used. From the calculations for this geometry [Fig. 3(a)], much of the underlying physics becomes more clear. While propagating through the sample, the fundamental spectrum becomes distorted due to spectral filtering, which leads to a lengthening of the pulse in time, and, thus to a reduction of the field amplitude and the Rabi frequency. This is the only way the high-energy transitions couple to the band gap nonlinearity. The result is a reduction of the splitting between the Rabi sidebands (compare Figs. 1 and 3). This explains the much smaller splitting seen in Fig. 3 of Ref. [14] as compared to the simple modeling; see Fig. 2 of Ref. [14]. If one uses laser pulses corresponding to the experiment rather than $\operatorname{sinc}^{2}$ pulses, this effect is even more pronounced. Because of the substantially reduced splitting, the influence on the CEO phase is expected to be largely reduced. Also, it becomes obvious from Fig. 3(a) of this Letter that the signal varies very strongly with propagation coordinate $z$. This is due to the fact that the absorption coefficients (for the third harmonic) of both GaAs and $\mathrm{Al}_{0.3} \mathrm{Ga}_{0.7}$ As are around $1 /(10 \mathrm{~nm})$ which can easily be estimated from the corresponding linear dielectric functions shown in Fig. 3(b). As a dramatic result, the detected signal does not stem from the $600 \mathrm{~nm}$ thick GaAs layer sandwiched between $\mathrm{Al}_{0.3} \mathrm{Ga}_{0.7}$ As barrierswhich was previously believed [14] - but rather from the thin GaAs cap layer initially employed as an antioxidation layer. Thus, in order to actually see effects of the CEO phase in resonant extreme nonlinear optics of the GaAs layer, one has to completely avoid any $\mathrm{Al}_{0.3} \mathrm{Ga}_{0.7} \mathrm{As}$ barriers as done above in (iii). Furthermore, it becomes clear that GaAs layer thicknesses much above some $10 \mathrm{~nm}$ do not increase the signal strength in the experiment.

In conclusion, we have pointed out the significant role of the CEO phase of few-cycle pulses in nonperturbative resonant nonlinear optics in a semiconductor as a result of the interference of different Rabi sidebands. In contrast to the corresponding known off-resonant perturbative effects, the dependence on the CEO phase is a function of the incident light intensity in this Letter-which highlights the difference between the two scenarios. If the intensity (the Rabi frequency) is calibrated, this novel effect might prove useful to determine the CEO phase of few-cycle laser pulses directly out of a mode-locked oscillator.

This cooperation is supported by the DFG. We thank H. Haug for stimulating discussions.

[1] T. Brabec and F. Krausz, Rev. Mod. Phys. 72, 545 (2000).

[2] P. Dietrich et al., Opt. Lett. 25, 16 (2000).

[3] A. V. Sokolov et al., Phys. Rev. Lett. 87, 033402 (2001).

[4] G. G. Paulus et al., Nature (London) 414, 182 (2001).

[5] D. J. Jones et al., Science 288, 635 (2000).

[6] A. Apolonski et al., Phys. Rev. Lett. 85, 740 (2000).

[7] U. Morgner et al., Phys. Rev. Lett. 86, 5462 (2001).

[8] M. Mehendale et al., Opt. Lett. 25, 1672 (2000).

[9] H. R. Telle et al., Appl. Phys. B 69, 327 (1999).

[10] C. G. Durfee III et al., Phys. Rev. Lett. 83, 2187 (1999).

[11] S. T. Cundiff et al., Rev. Sci. Instrum. 72, 3749 (2001).

[12] J. Reichert et al., Phys. Rev. Lett. 84, 3232 (2000).

[13] Th. Udem et al., Phys. Rev. Lett. 86, 4996 (2001).

[14] O. D. Mücke et al., Phys. Rev. Lett. 87, 057401 (2001).

[15] S. Hughes, Phys. Rev. Lett. 81, 3363 (1998).

[16] V. P. Kalosha et al., Phys. Rev. Lett. 83, 544 (1999).

[17] D. E. Aspnes et al., J. Appl. Phys. 60, 754 (1986).

[18] H. Haug and S.W. Koch, Quantum Theory of the Optical and Electronic Properties of Semiconductors (World Scientific, Singapore, 1993).

[19] H. Haug (private communication).

[20] O. D. Mücke et al., in "Quantum Coherence, Correlation and Decoherence in Semiconductor Nanostructures," edited by T. Takagahara (to be published).

[21] In general, GaAs also shows an off-resonant secondharmonic signal due to its lack of inversion symmetry. This (weak) signal can be suppressed by proper choice of the incident and detection light polarizations; for details, see X. Song, physics/0203023. 